\title{
Macrogeographic patterns of breeding system diversity in the Daphnia pulex group from the United States and Mexico
}

\author{
PAUL D. N. HEBERT* \& TERRIE L. FINSTON \\ Department of Zoology, University of Guelph, Guelph, Ontario, Canada N1G 2 W1
}

\begin{abstract}
Studies on the biogeographical patterning of reproductive systems promise to extend understanding of the factors which modulate breeding system transitions. Two closely allied cladoceran crustaceans, Daphnia pulex and D. pulicaria, show varied modes of reproduction, with populations reproducing by either cyclic or obligate parthenogenesis. Prior studies have provided a detailed understanding of their breeding system diversity in the polar and cold temperate regions of North America. The present investigation extends this analysis, characterizing breeding systems and clonal diversity at sites throughout the United States and Mexico. Genotypic diversity in these southern areas was high, but only diploids were detected, indicating that polyploids are restricted to the north. $\mathrm{F}_{1}$ hybrids and their two parental species were present in most areas, although their frequencies varied geographically. Hybrids invariably reproduced by obligate asexuality, but both parental taxa showed regional shifts in their breeding system. The complexity of these latter patterns suggests that they reflect the interplay of historical factors and selection.
\end{abstract}

Keywords: Cladocera, clone, Daphnia, North America, parthenogenesis, sexuality.

\section{Introduction}

Although sexual reproduction is dominant, asexually reproducing lineages appear to occur in all animal phyla (Stearns, 1987; Hines \& Culotta, 1998). Several models predict the environmental and ecological factors which might induce such variation in breeding system (see Bell, 1982 for review). However, efforts to critically evaluate the factors provoking transitions to asexuality have been constrained by the rarity of situations in which closely allied lineages differ solely in their mode of reproduction. In most cases, it is impossible to isolate the impacts of shifts to asexuality from the effects of ploidy level or heterozygosity variation which accompany transitions to this mode of reproduction. For example, since asexual vertebrates are invariably of hybrid origin (Dawley, 1989), appraisals of the impact of transitions to asexuality are confounded by the increased heterozygosity of asexuals relative to their sexual ancestors. In many other cases, identification of the effects of asexuality is complicated by the elevated ploidy levels which accompany the acquisition of asexuality.

\footnotetext{
*Correspondence. E-mail: phebert@uoguelph.ca
}

It has so far been possible to identify very few species which include some lineages reproducing sexually and others by parthenogenesis. However, two closely allied species of cladoceran crustaceans, Daphnia pulex and $D$. pulicaria, show variation in their breeding system, with some populations reproducing by cyclic parthenogenesis and others by obligate asexuality (Hebert et al., 1993). Lineages reproducing by cyclic parthenogenesis engage in regular bouts of sexual reproduction, while those employing obligate parthenogenesis have abandoned sexuality. Many of these obligately asexual clones are diploid and there is good evidence that they derive from a single parental species (Crease et al., 1989). The situation is not without complications; other asexual clones are of hybrid origin and some are polyploid (Beaton \& Hebert, 1988; Dufresne \& Hebert, 1994). Nonetheless the presence of cyclic and obligately asexual diploid clones of both species is well established through joint allozyme and DNA quantification studies (Beaton \& Hebert, 1988; Hebert et al., 1988, 1993).

Cyclic parthenogenesis is the ancestral breeding system of the genus Daphnia, as shown by its dominance among other cladoceran crustaceans (Taylor et al., 1999). Transitions to obligate asexuality have occurred rarely, so far being known in just one (pulex) of the 15 species complexes of Daphnia from North America 
(Colbourne et al., 1997). The occurrence of obligate asexuality in this complex has apparently been mediated by a sex limited meiosis suppressor which spread by introgression (Innes \& Hebert, 1988), so that four of the six species in this complex now include obligately asexual lineages. Obligately asexual populations of the D. pulex group dominate the polar regions of the entire Holarctic region (Weider et al., 1987, 1999). By contrast populations in the temperate regions of Europe seem invariably to reproduce by cyclic parthenogenesis (Ward et al., 1994; Palsson, 2000). Intensive studies on Canadian populations have revealed both the presence of obligately and cyclically parthenogenetic lineages and the regional patterning of breeding systems, perhaps reflecting the dispersal of populations from differing glacial refugia (Hebert et al., 1993). For example, populations of $D$. pulex in eastern Canada reproduce largely by obligate asexuality, whereas those in the west are cyclic parthenogens.

The present study extends the geographical scope of past investigations on breeding system variation in the D. pulex complex. It aimed, firstly, to determine if populations from the southern half of North America show the same breeding system diversity apparent in northern locales and to provide details on their genetic structure. For example, this investigation ascertains if polyploid clones occur on the southern periphery of the group's distribution as well as along its northern margins. It also attempts to clarify the role of climatic variables in modulating levels of clonal diversity in single populations by extending the geographical scale of investigation. Finally, the study aims to extend information on the patterning of breeding systems in this group to clarify the association between these transitions and environmental conditions.

\section{Materials and methods}

\section{Collections and electrophoresis}

Daphnia were collected from more than 2000 vernal ponds across the United States and Mexico from February to June of 1986-95 (Fig. 1). These habitats were sampled in early spring, shortly after their refill from winter rains or snow melt. Collections in the south (e.g. Mexico) were made in February, whereas those in the north (e.g. Montana) were made in early June. In a few areas in the western USA and Mexico where these habitats were rare or absent, small reservoirs and lakes were also sampled. This survey led to the collection of all 20 species of Daphnia known from pond habitats in this region (Hebert, 1995), but the present study only examines genotypic diversity in $D$. pulex, $D$. pulicaria and their interspecific hybrids. Members of this triad

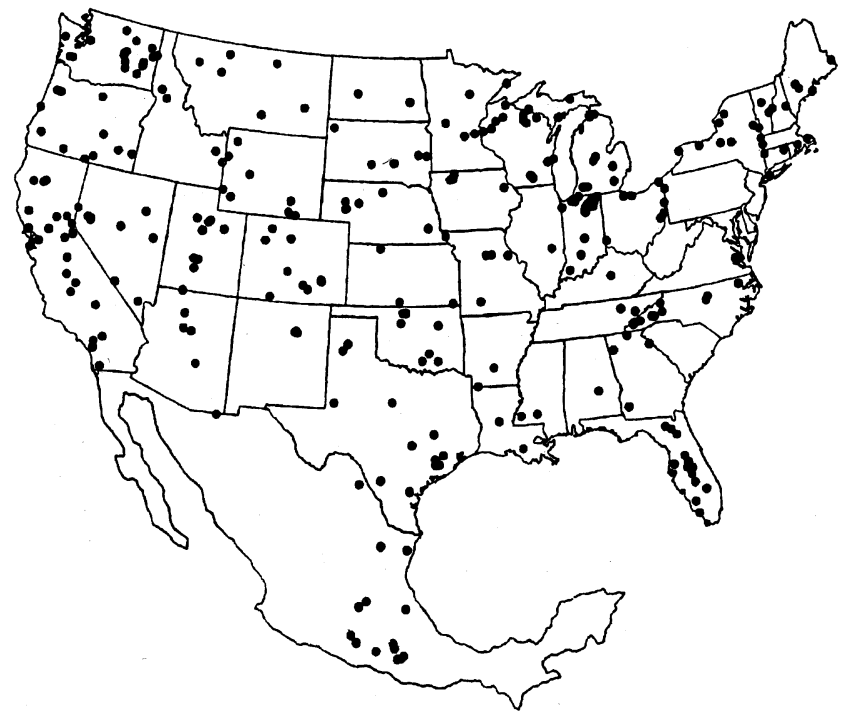

Fig. 1 Sampling sites for Daphnia in the United States and Mexico.

were sorted in the field and 50 individuals from each population were flash frozen in liquid nitrogen for later allozyme analysis. Although ponds were sampled in all 48 contiguous states, members of the triad were detected in only 426 habitats (412 ponds, 14 reservoirs/lakes) from 95 sites and 26 states (Table 1). When present, they were ordinarily the only daphniids present. To simplify the presentation of information, these populations have been assigned to one of seven geographical regions, comprising Mexico and six regions within the United States (Table 1).

Morphological discrimination of $D$. pulex and $D$. pulicaria is clouded by the prevalence of $F_{1}$ hybrids and by clonal variation in morphology, but these taxa can be recognized by an allelic substitution at lactate dehydrogenase (Hebert et al., 1989). In this study, individuals homozygous for the $L d h^{1.21}$ allele were classified as $D$. pulicaria, and $L d h^{1.00}$ homozygotes as D. pulex, whereas heterozygotes at this locus were classified as interspecific hybrids. Because of the large number of populations involved, mean allele frequencies were calculated for each species on a regional basis. The summary gene frequencies include data extracted from populations containing taxon mixtures.

Forty-four individuals from each population were analysed for variation at six other allozyme loci which are commonly polymorphic and well resolved (Hebert et al., 1993). These loci included supernatant aspartate aminotransferase (Aat-s), aldehyde oxidase (Ao), fumarate hydratase (Fumh), glucose-6-phosphate isomerase (Gpi), mannose phosphate isomerase (Mpi), and phosphoglucomutase (Pgm). Electrophoresis was carried out following standard procedures (Hebert \& 
Table 1 Seven regions of the United States and Mexico where Daphnia pulex, D. pulicaria or their $\mathrm{F}_{1}$ hybrids were detected. Numerals in parentheses indicate the number of habitats in each state where these taxa were found

\begin{tabular}{ll}
\hline Region & \multicolumn{1}{c}{ State } \\
\hline North-central (NC) & Idaho (19), Iowa (8), Minnesota (21), Montana (42), Nebraska (21), \\
North-east (NE) & North Dakota (18), South Dakota (12), Wyoming (17) \\
& Illinois (2), Maine (7), Michigan (68), Ohio (16), New York (33), \\
Vermont (1), Wisconsin (18) \\
North-west (NW) & Oregon (16), Washington (12) \\
South-central (SC) & Colorado (26), Kansas (1), New Mexico (5), Oklahoma (6) \\
South-east (SE) & Kentucky (1) \\
South-west (SW) & Arizona (17), California (13), Nevada (12), Utah (7) \\
Mexico (MX) & North Central (7) \\
\hline
\end{tabular}

Beaton, 1993) using Titan III cellulose acetate plates, and a Tris-glycine buffer system $(\mathrm{pH}=8.5)$.

Allelic variants were identified by their $\mathrm{R}_{\mathrm{f}}$ values, with their relative mobility reported in relation to the mobility of the gene product of a standard allele $\left(\mathrm{R}_{\mathrm{f}}=1.00\right)$ in a $D$. pulex clone (W2-8) from Windsor, Ontario. Because of the high diversity at $A o$, alleles at this locus were pooled into three mobility classes as in earlier studies (Hebert et al., 1993). All genetic analyses were carried out using BIOsYs-1 (Swofford \& Selander, 1989), except where noted. This paper reports $\mathrm{R}_{\mathrm{f}}$ values for allelic variants, in contrast to letter codes employed in earlier studies. However, the dominant alleles are the same as those detected in prior work (e.g. Hebert et al., 1993), enabling comparison of the data sets. All populations were screened for the presence of allozyme phenotypes that are characteristic of polyploids, including 'unbalanced' heterozygotes and those with extra (i.e. 3 or 4 ) zones of activity at loci which produce monomeric enzymes.

\section{Breeding system diagnosis}

Because of the scale of this study, it was not possible to carry out breeding studies on each population. Instead breeding systems were diagnosed from inspection of genotypic arrays. The criteria employed in this diagnosis were developed in earlier investigations and were validated using breeding studies to test the mode of reproduction of populations which were initially determined from genotypic surveys (Hebert \& Crease, 1983; Innes \& Hebert, 1988; Innes et al., 2000). This earlier work identified two factors that can impede a correct diagnosis - habitat permanence and small sample size. The analysis of populations from permanent habitats is complicated because long bouts of parthenogenesis can conceal the ability of the resident taxa to reproduce sexually. Similarly the effort to determine breeding systems from a small sample size runs the risk of misdiagnosis because of sampling error.

Our study was protected against these sources of breeding system misassignments in two ways. Firstly, we focused our work on vernal pools, sampling populations shortly after their re-establishment when the genotypic structure of cyclic parthenogens should approximate those in sexual lineages. We also protected our results against the effects of low sample size by analysing 44 individuals from each population and only making a diagnosis for the most abundant taxon. As $79.8 \%$ of the habitats contained only a single member of the triad and most other sites were dominated by one taxon, this was not a serious constraint.

The present analysis based breeding system decisions on the same genotypic criteria employed in earlier studies on the D. pulex complex (Hebert et al., 1988). Briefly, three parameters were used to diagnose the breeding system of any population which showed polymorphism at one or more loci: the number of unique multilocus genotypes (NCLONES); the probability of the observed genotypic array conforming to Hardy-Weinberg (HW) expectations (LOGP), calculated by averaging the log probability values of the observed genotypic arrays at each polymorphic locus; and the genotypic diversity ratio (GDR), the number of observed multilocus genotypes divided by the number expected in a sample of the same size from a sexual population with the same gene frequencies using 100 Monte Carlo simulations. The GDR approximates unity in populations showing conformance to Hardy-Weinberg expectations and linkage equilibrium. A locus was considered polymorphic if the frequency of the most common allele did not exceed 0.95 .

Populations that were monomorphic at all six loci were presumed to reproduce via cyclic parthenogenesis, whereas the breeding systems of populations polymorphic at one or more loci were diagnosed using the following rules. Populations were classified as 
obligately asexual when the probability that genotypic frequencies were in $\mathrm{HW}$ equilibrium was less than $1 \%$ (i.e. LOGP was <-2.00). In these populations, the GDR also tended to be low (generally <0.50) and NCLONES was small. Populations were classified as reproducing by cyclic parthenogenesis when LOGP was greater than -2.00 and the GDR was greater than 0.75 . The breeding systems of populations which combined a LOGP greater than -2.00 with a GDR of $<0.75$ were not diagnosed.

\section{Results}

\section{Taxon distributions}

Daphnia pulex was the most abundant taxon in the United States and Mexico, comprising $60 \%$ of the 19638 individuals that were analysed, whereas D. pulicaria and the $F_{1}$ hybrids totalled $13 \%$ and $27 \%$, respectively. Although members of the triad were present in all seven regions, they were rare in the SE and MX (Table 2). $\mathrm{F}_{1}$ hybrids co-occurred with one of their parent species in $17.4 \%$, and with both parents in $2.8 \%$ of the habitats where the triad was present (Table 2). Most habitats contained only one taxon; D. pulex occurred alone in $44.4 \%, \mathrm{~F}_{1}$ hybrids in $23.7 \%$ and D. pulicaria in $11.7 \%$ of the habitats. The relative frequencies of these three taxa showed conspicuous geographical variation, with $D$. pulicaria dominant in the west and D. pulex in the north-east (Fig. 2). In the southern USA, D. pulex only occurred in montane regions with substantial winter snowfalls, whereas D. pulicaria and hybrid clones were more widely distributed. Hybrid clones were present in approximately $40 \%$ of the habitats in most areas, but were dominant in the SE and MX.

\section{Breeding system assignments}

Of the 426 populations analysed, 379 were polymorphic at one or more loci, whereas the remaining 47 were monomorphic at all loci. There was no evidence of polyploid genotypes; all heterozygotes showed allozyme phenotypes typical of disomic inheritance. Variation in the three parameters used to characterize populations was considerable (Fig. 3). For example, the number of unique multilocus genotypes per population ranged from 1 to 41, while the GDR ranged from 0.03 to 1.49. The incidence of populations whose breeding system could not be assigned varied among regions. It was lowest in the NC $(6.3 \%)$, SC $(7.1 \%)$ and $\mathrm{NE}(7.1 \%)$ regions; higher in the NW $(15.3 \%)$ and highest in the SW and Mexico (33.3\%). The rise in undiagnosed populations in the last regions coincided with the survey of permanent habitats where reproduction via sustained parthenogenesis was likely. Within the subset of populations designated as reproducing by obligate parthenogenesis, clonal diversity varied geographically (Table 3 ), with the highest levels in the SW for $D$. pulex and in the NW for $D$. pulicaria. The lowest levels of diversity were detected in the $\mathrm{SC}$ region for both species.

The genotypic characterizations enabled the diagnosis of breeding systems for $D$. pulicaria and $D$. pulex from 14 and 20 states, respectively. Populations of $D$. pulex reproducing by obligate asexuality were restricted to the southern and north-eastern margins of its range (Fig. 4a). Many states sited in the central and northwestern United States included solely cyclic parthenogens, whereas populations with varied breeding systems occurred primarily in the mid-west. D. pulicaria showed a lower incidence of cyclic parthenogens with only a single state (Washington) dominated by populations of this type (Fig. 4b). Sites showing mixed breeding systems

Table 2 Proportions of habitats in Mexico and six regions of the United States containing Daphnia pulex, D. pulicaria, $\mathrm{F}_{1}$ hybrids or mixtures of these taxa. $N$, number of habitats with members of the triad

\begin{tabular}{|c|c|c|c|c|c|c|c|c|}
\hline \multirow[b]{3}{*}{ Taxonomic composition } & \multirow[b]{3}{*}{$N$} & \multicolumn{7}{|c|}{ Geographical regions } \\
\hline & & NW & $\mathrm{NC}$ & $\mathrm{NE}$ & SW & $\mathrm{SC}$ & $\mathrm{SE}$ & MX \\
\hline & & 28 & 158 & 145 & 49 & 38 & 1 & 7 \\
\hline \multicolumn{9}{|l|}{ Single species: } \\
\hline D. pulex & & 0.07 & 0.33 & 0.79 & 0.26 & 0.18 & - & - \\
\hline $\mathrm{F}_{1}$ hybrids & & 0.21 & 0.32 & 0.06 & 0.31 & 0.34 & 1.0 & 0.86 \\
\hline \multicolumn{9}{|l|}{ Taxon mixtures: } \\
\hline $\mathrm{F}_{1}+D$. pulex & & 0.04 & 0.11 & 0.14 & 0.08 & 0.11 & - & - \\
\hline $\mathrm{F}_{1}+D$. pulicaria & & 0.07 & 0.10 & 0.01 & 0.02 & 0.16 & - & 0.14 \\
\hline D. pulex + D. pulicaria & & 0.04 & - & - & - & - & - & - \\
\hline All three taxa & & 0.07 & 0.06 & - & - & 0.03 & - & - \\
\hline
\end{tabular}


Fig. 2 Pie diagrams showing the proportions of Daphnia pulex, D. pulicaria, and their $F_{1}$ hybrids in the United States and Mexico.

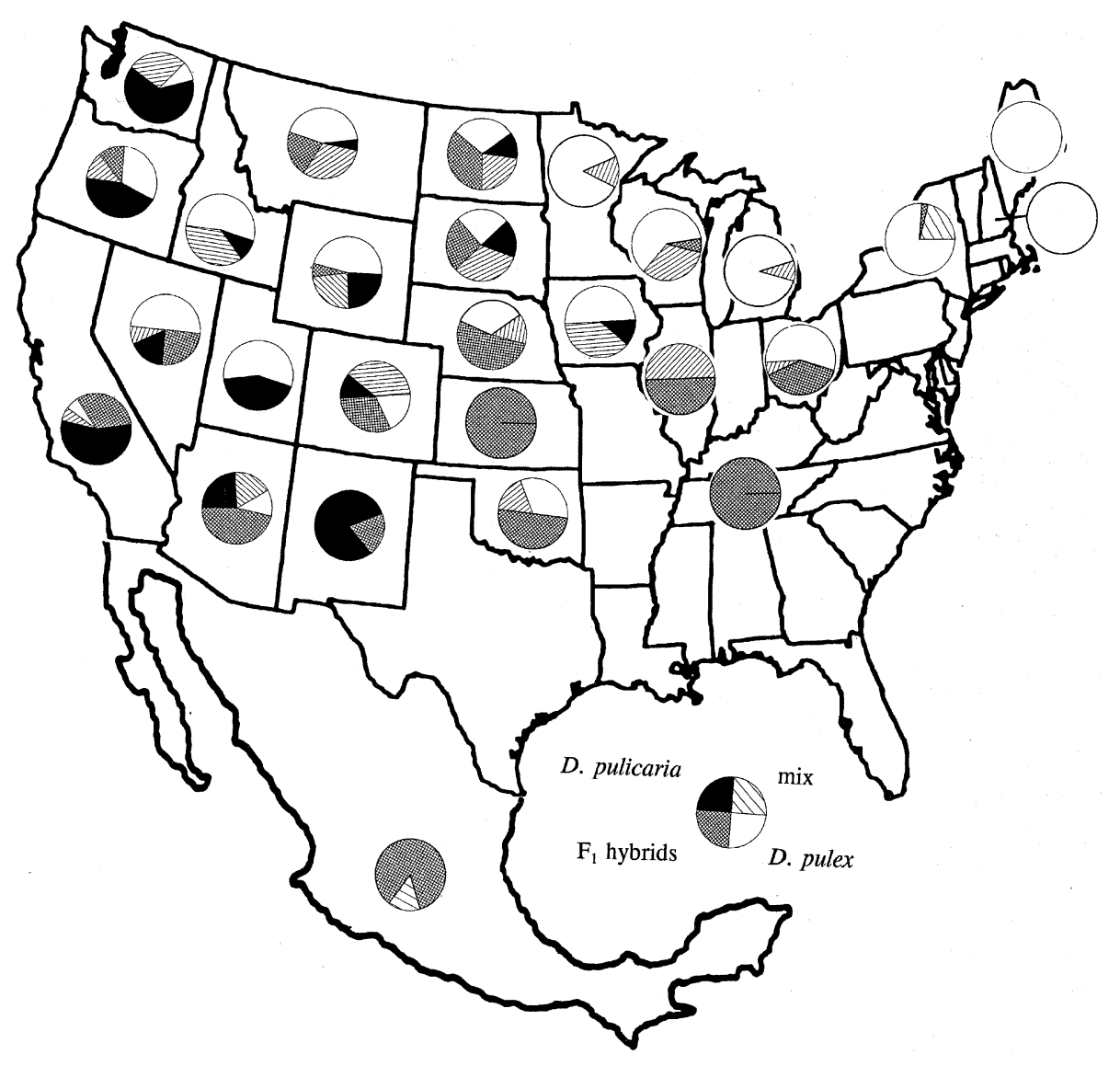

were largely restricted to the south-west, whereas obligately parthenogenetic lineages dominated the mid-west.

\section{Discussion}

Past work has shown that the arctic regions of Eurasia and North America are occupied by obligately asexual lineages of the D. pulex complex (Weider et al., 1999). Habitats in these areas are usually dominated by polar endemics, such as D. middendorffiana or D. tenebrosa, but $D$. pulicaria is also present. By contrast, the prairie as well as the boreal and deciduous forest regions of Canada are dominated by D. pulex, D. pulicaria and their $F_{1}$ hybrids (Hebert et al., 1993). In the forested regions of eastern Canada, D. pulicaria is restricted to lakes, while $D$. pulex and $\mathrm{F}_{1}$ hybrids occur in temporary ponds. In western Canada, all three taxa occur in ponds, but $D$. pulicaria retains its dominance in lakes. The present investigation indicates that this same triad dominates ponds in the United States and Mexico. Moreover, the distributional pattern of the parental taxa in this region reinforces that seen in Canada. D. pulex dominated ponds in the forested north-east, but $F_{1}$ hybrids were also present. By contrast, in the central and western USA all three taxa occurred in ponds. Fourteen of the habitats surveyed in this latter region were lakes rather than ponds and, excepting two Mexican habitats dominated by hybrids, $D$. pulicaria was the sole taxon detected. The absence of members of the triad from ponds in the SE reflected their replacement by D. obtusa, a species with a greater tolerance of high temperatures (MacIsaac et al., 1985).

$\mathrm{F}_{1}$ hybrids between $D$. pulex and $D$. pulicaria were common at sites across North America, representing $27 \%$ of the isolates from the United States and Mexico and $22 \%$ in the temperate regions of Canada. As past breeding studies have shown that these hybrids invariably reproduce by obligate parthenogenesis (Hebert \& Crease, 1983; Hebert et al., 1993), these clones can extend their distribution beyond their point of origin through the dispersal of asexual ephippial eggs. The demonstration of such long-range transport is complicated in most regions of North America by the presence of both parental species. However, the detection of seven hybrid clones at sites in the SE and MX, where neither parent was present, confirms the importance of such dispersal events. It does not appear, however, that long-range dispersal leads to a flood of migrants, because hybrid clones in other areas have genotypic arrays reflecting local variation in the gene frequencies of their parental taxa. In addition, 

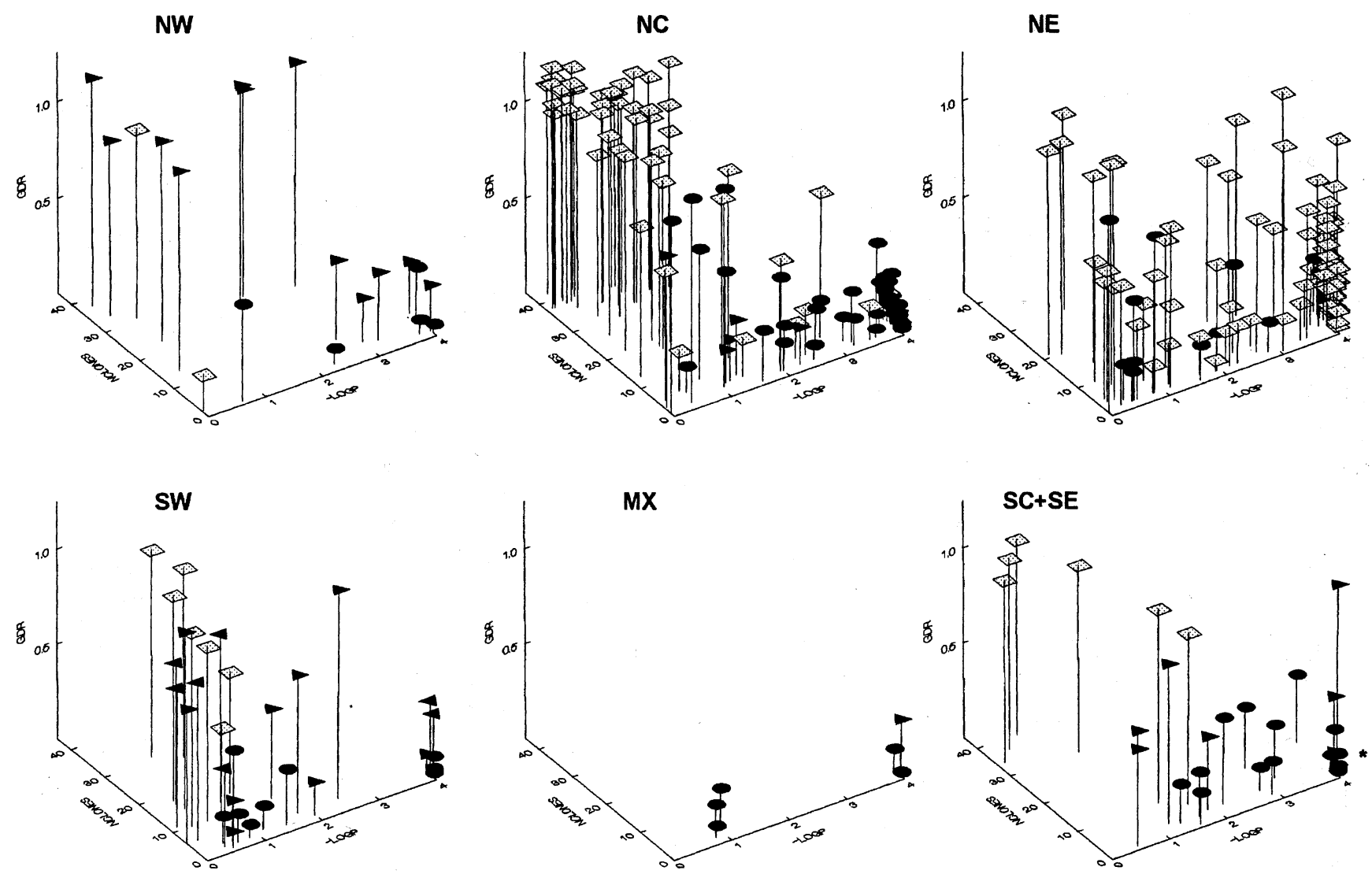

Fig. 3 Genotypic characteristics for populations of Daphnia pulex (squares), D. pulicaria (filled triangles), and $\mathrm{F}_{1}$ hybrids (filled circles). Populations of $D$. pulicaria from lakes or reservoirs are represented by triangles with their apex to the left, while those from ponds have their apex to the right. The sole population from the SE region is marked with an asterisk.

hybrids were both more clonally diverse and abundant in the west, where the co-occurrence of $D$. pulex and D. pulicaria in single habitats was commonest, suggesting increased opportunity for their synthesis.

Whereas past studies have shown that polyploid clones are common in populations of the pulex complex from tundra regions (Beaton \& Hebert, 1988; Dufresne \& Hebert, 1994, 1997; Ward et al., 1994), only diploids are known from the temperate zone of Canada. The present analysis has established that polyploids are also absent from the USA and Mexico, suggesting that polyploids in this group are restricted to low-temperature rather than extreme environments. This pattern appears to have some generality as polyploid lineages of a second cladoceran genus, Bosmina, are also restricted to the north (Little et al., 1997).

Obligately asexual lineages of daphniids are remarkable for their high regional diversity. For example, thousands of obligately asexual clones of $D$. pulex occur in the Great Lakes watershed (Hebert et al., 1988). Although regional variation is immense, clonal variation in single populations is restrained and relatively inflexible. Obligately asexual populations in the $D$. pulex complex possess an average of 4.5 clones in the arctic (Weider et al., 1999) vs. 2.1 clones in the temperate regions of Canada (Hebert et al., 1993). A similar level of diversity was detected in this study, with 2.9 clones/ population for D. pulex and 3.6 clones/population for $D$. pulicaria. The fact that local diversity is so restrained is significant in three regards. Firstly, given the large number of clones present in each region, the low diversity of local assemblages suggests that selection has set an upper limit, with new arrivals either displacing resident taxa or failing to become established. Moreover, despite variation in taxon composition, ploidy levels and climatic regime, the equilibrium diversity in populations of these crustaceans only varies from 2 to 4 clones. By contrast, much higher levels of clonal diversity have been reported in asexual ostracodes which occupy the same habitats but are benthic (Havel \& Hebert, 1989). If this difference between planktonic and benthic asexuals is general, it would suggest that the structural complexity of habitats plays an important role in setting an upper limit to clonal diversity. 
Table 3 Variation in clonal diversity among obligately asexual populations of Daphnia pulex, D. pulicaria and their $\mathrm{F}_{1}$ hybrids from six regions in the United States and Mexico. $N$, number of populations

Geographical regions

\begin{tabular}{|c|c|c|c|c|c|c|c|c|}
\hline \multirow{2}{*}{$\begin{array}{l}\text { Species or } \\
\text { hybrids }\end{array}$} & & \multirow[b]{2}{*}{ NW } & & & & & \multirow[b]{2}{*}{ SE } & \\
\hline & & & $\mathrm{NC}$ & $\mathrm{NE}$ & SW & $\mathrm{SC}$ & & MX \\
\hline \multirow[t]{4}{*}{ D. pulex } & $N$ & 1 & 9 & 78 & 1 & 1 & - & - \\
\hline & $\bar{x}$ & 2.0 & 3.4 & 2.8 & 9.0 & 1.0 & - & - \\
\hline & SD & - & 1.6 & 1.6 & - & - & - & - \\
\hline & Range & 2 & $1-6$ & $1-9$ & 9 & 1 & - & - \\
\hline \multirow[t]{4}{*}{ D. pulicaria } & $N$ & 5 & 11 & - & 5 & 5 & - & \\
\hline & $\bar{x}$ & 5.4 & 3.1 & - & 4.2 & 2.4 & - & \\
\hline & SD & 2.6 & 1.5 & - & 2.0 & 1.0 & - & \\
\hline & Range & $2-8$ & $1-5$ & - & $2-10$ & $1-4$ & - & \\
\hline \multirow[t]{4}{*}{$\mathrm{F}_{1}$ hybrids } & $N$ & 8 & 60 & 25 & 15 & 22 & 1 & 6 \\
\hline & $\bar{x}$ & 2.0 & 3.5 & 2.4 & 1.7 & 2.9 & 1.0 & 2.3 \\
\hline & SD & 1.7 & 1.8 & 1.8 & 0.8 & 3.3 & - & 1.2 \\
\hline & Range & $1-6$ & $1-8$ & $1-8$ & $1-3$ & $1-13$ & 1 & $1-4$ \\
\hline
\end{tabular}

Although niche complexity may explain this divergence in clonal diversity between populations of ostracodes and daphniids, other factors appear to modulate local diversity. For despite the regional congruence in mean clonal diversity, individual asexual populations of Daphnia contained from 1 to 13 clones. Habitats with a single clone may reflect competitive exclusion, a result commonly observed in laboratory microcosm studies of daphniids (Loaring \& Hebert, 1981), but populations with high diversity are less easily explained. Some of these cases are probably artefactual, representing populations with mixed breeding systems. Cases of such misdiagnosis, which can only be resolved by breeding studies, arise when a population dominated by one or two asexual clones co-occurs with a low incidence of cyclic parthenogens. However, other cases of co-occurrence cannot have this explanation. For example, populations consisting solely of $F_{1}$ hybrids contained a peak of 6-13 clones in various regions of the United States. There is clearly a need for detailed studies of clonal interactions in these settings.

The present study has employed the genotypic attributes of populations to diagnose their breeding systems. The validity of this approach has been confirmed by earlier investigations which combined this diagnostic approach with subsequent breeding studies (Innes \& Hebert, 1988; Innes et al., 2000). This work has shown that the diagnosis of breeding systems is least secure in permanent bodies of water where populations capable of sex may reproduce by sustained parthenogenesis. In these circumstances, diversity can decay, resulting in populations whose genotypic composition deviates markedly from those expected under sexual reproduction. However, such divergence is not inevit- able, as lake populations of $D$. pulicaria often possess genotypic frequencies which approximate those expected in a sexual population (Cerny \& Hebert, 1993). The present study largely escaped this interpretational complexity by focusing analysis on vernal ponds. A small percentage $(3 \%)$ of the habitats were permanent, but D. pulicaria was ordinarily the only species in these settings and most populations were diagnosed as cyclic parthenogens. The only exceptions involved two Mexican reservoirs which were dominated by hybrids.

The present results, when coupled with earlier investigations, provide a continental perspective on breeding system diversity in D. pulex and D. pulicaria (Fig. 4). The results on these two species are, in one sense, consistent; their variation in breeding systems shows no simple linkage to major biomes or to surrogates of climatic variation such as latitude. Populations of $D$. pulex reproducing by cyclic parthenogenesis do dominate the grassland biome, but they also occur as exceptions in three other biomes (boreal forest, deciduous forest, desert). There is an indication that cyclically parthenogenetic populations in this species are encircled by obligate asexuals along their southern, eastern and northern range margins. If further work in the north confirms this pattern, it would suggest that transitions to asexuality are favoured by extreme environments or that asexuals are on the leading edge of range extension. The patterning of breeding systems in D. pulicaria is less obvious, but there is an indication that obligate asexuals dominate the eastern margins of its range and cyclics the west. These regional patterns of breeding system variation might reflect the origin and subsequent diffusion of asexuality from specific areas of North America. Populations with 'misplaced' breeding systems might 


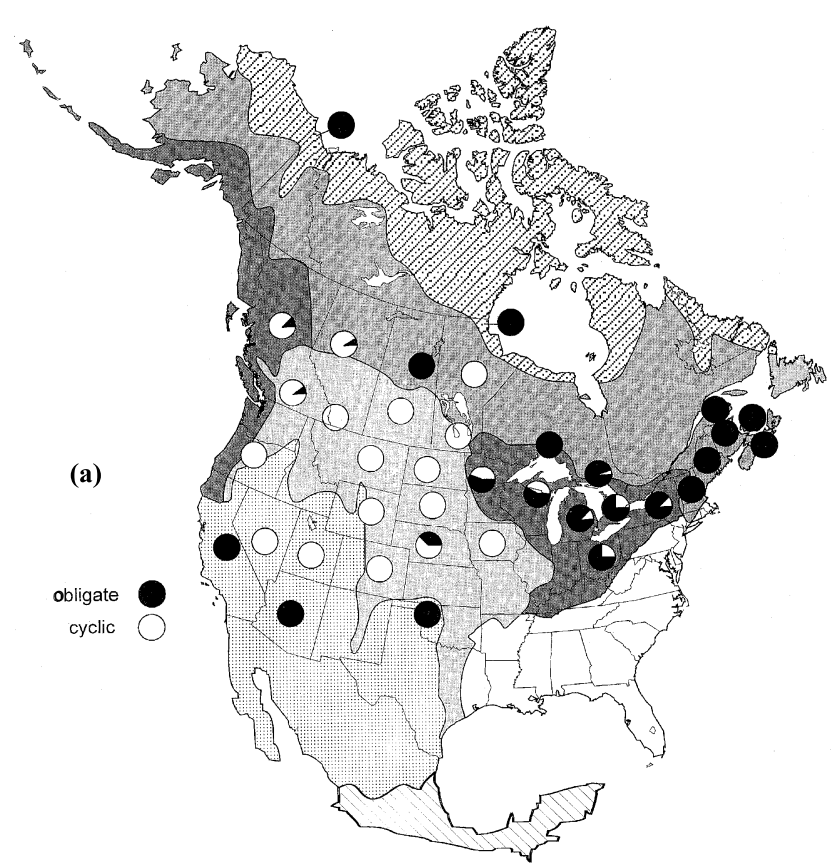

Fig. 4 Breeding system designations for (a) populations of Daphnia pulex and (b) D. pulicaria in the major biomes of North America. Data for United States and Mexico are from this study, whereas breeding system designations for Canadian populations are based on prior studies (Hebert et al., 1993). Populations whose breeding system could not be diagnosed were excluded from this plot.

simply reflect long-distance dispersal from areas which supported populations with divergent breeding systems during the Pleistocene. If historical factors have played a dominant role in determining the modern distributions of breeding systems, one would expect other signs of this fact, such as mtDNA divergence between populations with different breeding systems. If, on the other hand, breeding system adjustments represent local adaptations driven by selection, no covariation between breeding systems and $\mathrm{mt}$ diversity should be apparent. The fact that nearly $30 \%$ of sites with D. pulex and $40 \%$ of those with $D$. pulicaria possess populations with varied breeding systems provides a direct opportunity to investigate this issue.

This study has provided an overview of the genotypic attributes of the $D$. pulex complex on a continental scale. It is now apparent that polyploid clones are restricted to the north. It is also evident that the average clonal diversity in asexual populations in this group is remarkably congruent across broad climatic gradients, but subject to marked variation among local populations. Finally the general patterning of breeding system diversity is clear. The challenge for the future lies in studies which probe the factors responsible for generating these patterns of variation.

\section{Acknowledgements}

This research was supported by several NSERC grants to PDNH. We thank S. Schwartz, R. DeMelo, D. Taylor, J. Turgeon, R. Allison, and P. Gadja for help with the field work, and J. Overton and R. Allison for assistance with data analysis. Comments by T. Crease, D. Taylor and J. Colbourne improved the manuscript.

\section{References}

BEATON, M. J. AND HEBERT, P. D. N. 1988. Geographical parthenogenesis and polyploidy in Daphnia pulex Leydig. Am. Nat., 132, 837-845.

BELL, G. 1982. The Masterpiece of Nature: The Evolution and Genetics of Sexuality. Croom Helm, London.

(c) The Genetics Society of Great Britain, Heredity, 87, 153-161. 
CERNY, M. AND HEBERT, P. D. N. 1993. Genetic diversity and breeding system variation in Daphnia pulicaria from North American lakes. Heredity, 71, 497-507.

COlbourne, J. K., HEBERT, P. D. N. AND TAYLOR, D. J. 1997. Evolutionary origins of phenotypic diversity in Daphnia. In: Givnish, T. T. and Systema, K. (eds) Molecular Evolution and Adaptive Radiations. Cambridge University Press, Cambridge.

CREASE, T. J., STANTON, D. J. AND HEBERT, P. D. N. 1989. Polyphyletic origins of asexuality in Daphnia pulex. II. Mitochondrial DNA variation. Evolution, 43, 1016-1026.

DAWLEY, R. M. 1989. An introduction to unisexual vertebrates. In: Dawley, R. M. and Bogart, J. (eds) Evolution and Ecology of Asexual Vertebrates. Bulletin 466, New York State Museum, Albany, NY.

DUFRESNE, F. AND HEBERT, P. D. N. 1994. Hybridization and the origins of polyploidy. Proc. Roy. Soc. B, 258, 141-146.

DUFRESNE, F. AND HEBERT, P. D. N. 1997. Pleistocene glaciations and polyphyletic origins of polyploidy in an arctic cladoceran. Proc. Roy. Soc. B, 264, 201-206.

HAVEL, J. E. AND HEBERT, P. D. N. 1989. Apomictic parthenogenesis and genotypic diversity in Cypridopsis vidua (Ostracoda: Cyprididae). Heredity, 62, 383-392.

HEBERT, P. D. N. 1995. The Daphnia of North America. CD-ROM. University of Guelph.

HEBERT, P. D. N. AND BEATON, M. J. 1993. Methodologies for Allozyme Analysis Using Cellulose Acetate Electrophoresis, 2nd edn. Helena Laboratories, Beaumont, TX.

HEBERT, P. D. N. AND CREASE, T. 1983. Clonal diversity in populations of Daphnia pulex reproducing by obligate parthenogenesis. Heredity, 51, 353-369.

HEBERT, P. D. N., WARD, R. D. AND WEIDER, L. J. 1988. Clonal diversity patterns and breeding system variation in Daphnia pulex: an asexual-sexual complex. Evolution, 42, 147-159.

HEBERT, P.D. N., BEATON, M. J., SCHWARTZ, S. S. AND STANTON, D. J. 1989. Polyphyletic origins of asexuality in Daphnia pulex. I. Breeding system variation and levels of clonal diversity. Evolution, 43, 1004-1015.

HEBERT, P. D. N., SCHWARTZ, S. S., WARD, R. D. AND FINSTON, T. L. 1993. Macrogeographic patterns of breeding system variation in the Daphnia pulex group. I. Breeding systems of Canadian populations. Heredity, 70, 148-161.
HINES, P. AND CUlOTTA, E. 1998. The evolution of sex. Science, 281, 1979-2008.

INNES, D. J. AND HEBERT, P. D. N. 1988. The origin and genetic basis of obligate parthenogenesis in Daphnia pulex. Evolution, 42, 1024-1035.

INNES, D. J., FOX, C. J. AND wINSOR, G. L. 2000. Avoiding the cost of males in obligately asexual Daphnia pulex (Leydig). Proc. Roy Soc. B, 267, 991-999.

LitTle, T. J., DE MELO, R., TAYLOR, D. J. AND HEBERT, P. D. N. 1997. Genetic characterization of an arctic zooplanker: insights into geographic polyploidy. Proc. Roy Soc. B, 264, 1363-1370.

LOARING, J. M. AND HEBERT, P. D. N. 1981. Ecological differences among clones of Daphnia pulex (Leydig). Oecologia, 51, 162-168.

MACISAAC, H. J., HEBERT, P. D. N. AND SCHWARTZ, S. S. 1985. Inter and intraspecific variation in acute thermal tolerance of Daphnia. Physiol. Zool., 58, 350-355.

PALSSON, S. 2000. Microsatellite variation in Daphnia pulex from both sides of the Baltic Sea. Mol. Ecol., 9, 1075-1089.

STEARNS, S. C. 1987. The Evolution of Sex and its Consequences. Birkhäuser Verlag, Basel.

SWOFFORD, D. L. AND SELANDER, R. B. 1989. BIOSYS-1. A computer program for the analysis of allelic variation in population genetics and biochemical systematics. Release 1.7. Illinois Natural History Survey, Urbana, IL.

TAYLOR, D. J., CREASE, T. J. AND BROWN, W. M. 1999. Phylogenetic evidence for a single long-lived clade of crustacean cyclic parthenogens and its implications for the evolution of sex. Proc. Roy Soc. B, 266, 791-799.

WARD, R. D., BICKERTON, M. A., FINSTON, T. L. AND HEBERT, P.D. N. 1994. A geographic cline in breeding systems and ploidy levels in European populations of Daphnia pulex. Heredity, 73, 532-543.

WEIDER, L. J., BEATON, M. J. AND HEBERT, P. D. N. 1987. Clonal diversity in high-arctic populations of Daphnia pulex, a polyploid apomictic complex. Evolution, 41, 1335-1346.

WEIDER, L. J., HOBAEK, A., HEBERT, P. D. N. AND CREASE, T. J. 1999. Circumarctic phylogeography of an asexual species. II. Allozymic variation and clonal structure in arctic Daphnia. Mol. Ecol., 8, 1-13. 